\title{
Super bug colonizations rise at McGill University Health Centre
}

Published at www.cmaj.ca on June 29

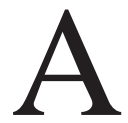
ggressive screening procedures at the McGill University Health Centre in Montréal, Quebec, have revealed an increase in the number of patients contaminated with vancomycin-resistant enterococci (VRE) over the last two years.

Although the centre is stopping short of declaring a full-fledged outbreak, its infection prevention and control service continues to find new colonizations of the antibiotic-resistant bacteria as they expand their screening profile.

"As soon as we started screening, the trend was obvious," says Dr. Charles Frenette, medical director of the service. "We've had sort of mini-outbreaks on different units and so far, we've been successful in controlling them.

The enterococci bacteria are part of the normal flora of the intestines, but can sometimes causes infections. Those infections are typically treated with antibiotics, like vancomycin. In the last two decades, virulent strains of antibiotic-resistant enterococci have emerged in hospitals. While VRE may inhabit a host and cause no discernable health problems, colonized individuals are at an increased risk of developing VRE infections, which are difficult to treat and can be fatal.

Representatives for the McGill University Health Centre won't say how many cases of VRE colonization have been identified over the last two years, or what percentage of increase those cases represent.

As for infections, Frenette says there's been a slight increase in tandem with the increase in cases of colonization. "So far this year, we're talking maybe 10 infections across four hospitals. These aren't big numbers."

The infection rate of VRE-colonized patients can be less than $1 \%$, or up to $5 \%$, says Frenette. "That's roughly the rapport

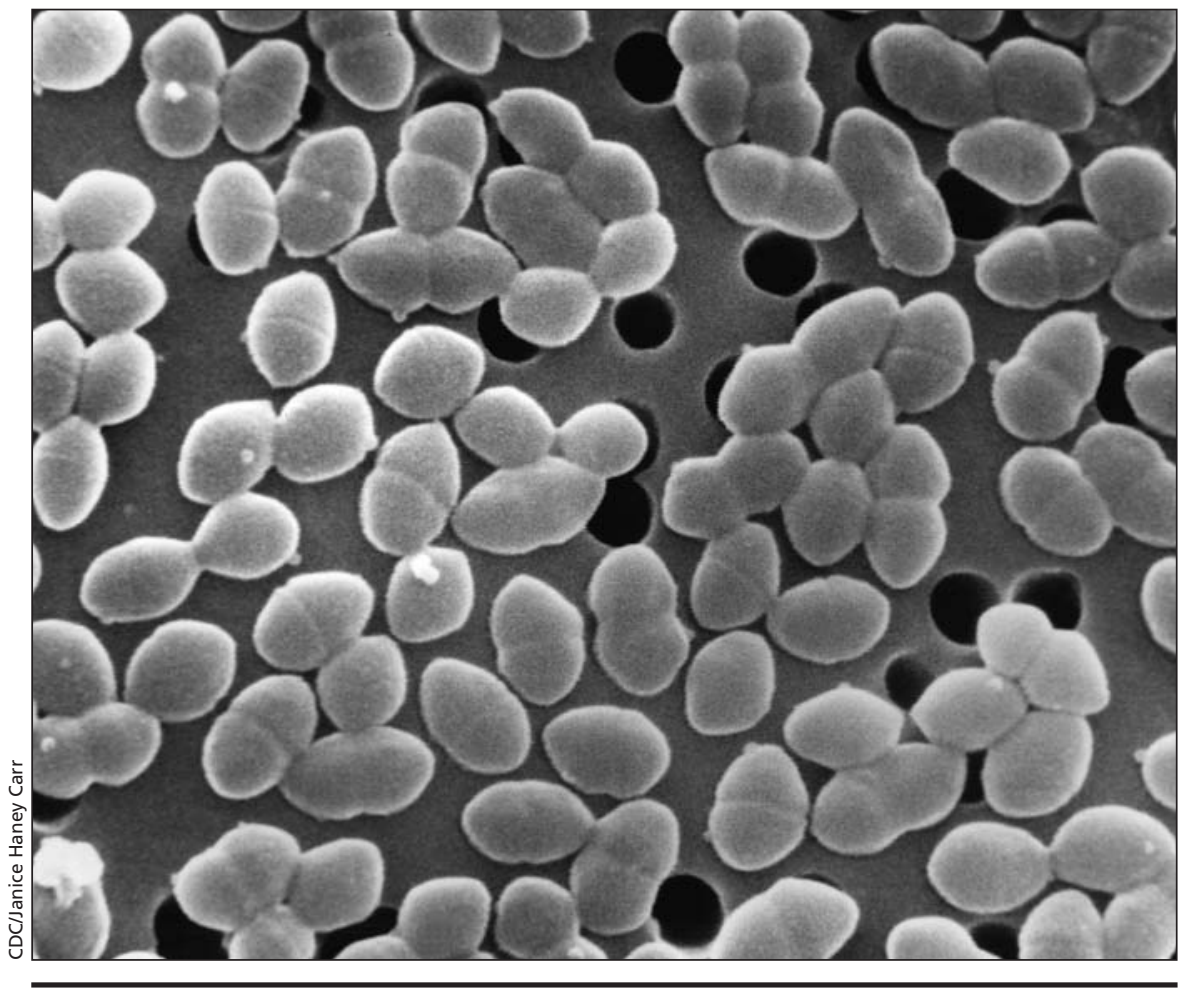

Enterococcus spp. bacteria have been linked as etiologic agents for hospital-borne illnesses such as vancomycin-resistant enterococci infections.

we've been seeing. It depends very much on the population affected by VRE.'"

That means that between January and May, the centre may have seen anywhere between 200 and more than 1000 new cases of VRE colonization.

“The main reason we're seeing more cases is that we're doing more screening than we used to," says Frenette. "We used to do maybe 10000 to 15000 screens a year. Now, we're up to 30000 or 40000 a year."

Under the centre's screening policy, every new case of VRE detected by the infection protection and control service entails weekly screening until there are no more cases transmitted on the unit, he says. "We're constantly looking for a reservoir of cases so we can prevent further transmission. We'd screen one floor, and it would lead to another floor and another, and so on."
Another reason the centre has a higher risk of VRE contamination is the types of procedures it provides and the type of patient it cares for.

"We're the only centre in Quebec that has solid organ transplants, bone marrow transplants, cardiac surgery, heavy hematology-oncology, two large dialysis units and a major trauma centre, a very large hepatobiliary unit, in addition to seven intensive care units. These subspecialties contribute significantly to the number of colonizations," Frenette explains. "The patients are sicker, are often immunosuppressed, and receive a wide variety of antibiotics and invasive procedures; all of which rend[er] them at risk for VRE colonization and infection."

The centre's use of sensitive diagnostic tools in VRE screening procedures may also have something to do with the 
higher number of colonization cases being detected, says Frenette. "We use polymerase chain reaction screening, which is more rapid but also $20 \%$ more sensitive than the traditional methods used by most hospitals to detect VRE."

In Canada, policies and procedures for VRE screening vary widely from hospital to hospital, making national comparisons and surveillance of the super bug difficult.

"No two hospitals have the same approach. Some screen only intensive care patients, others only screen for certain risk factors, and some hospitals won't do any screening whatsoever, as they may not have patients at risk for infections," says Frenette. "The bottom line is, if you don't screen, you won't find anything."

If a hospital's VRE rate is based solely on clinical cultures from infected patients, the facility may be adequately reporting its infection rate, but runs the danger of underestimating the potential transmissibility of VRE within that facility. Screening for colonized patients allows for infection control measures to be implemented, reducing transmission and the risk of infection.

At the McGill University Health Centre, those measures include isolating patients colonized with VRE in pri- vate rooms, requiring that anyone entering those rooms to wear a gown and gloves, and sanitizing the room after the patient is discharged.

"On floors where we've intervened, we've seen decreases in transmission, and generally we've had very few infections," says Frenette.

Frenette says the number of new VRE cases will likely continue to rise in the coming months, as the centre further expands its screening profile. "I expect that it will probably take six to 12 more months for VRE cases to start decreasing." - Lauren Vogel, CMAJ

DOI:10.1503/cmaj.109-3305 